Bull. Korean Math. Soc. 46 (2009), No. 4, pp. 691-700

DOI 10.4134/BKMS.2009.46.4.691

\title{
EXISTENCE OF SOLUTIONS OF QUASILINEAR INTEGRODIFFERENTIAL EVOLUTION EQUATIONS IN BANACH SPACES
}

\author{
Krishnan Balachandran and Dong Gun Park
}

\begin{abstract}
We prove the local existence of classical solutions of quasilinear integrodifferential equations in Banach spaces. The results are obtained by using fractional powers of operators and the Schauder fixedpoint theorem. An example is provided to illustrate the theory.
\end{abstract}

\section{Introduction}

The problem of existence of solutions of quasilinear evolution equations in Banach spaces has been studied by many authors [1, 2, 5-7, 12, 15-24, 26]. Crandall and Souganidis [8] have proved the existence, uniqueness and continuous dependence of a continuously differentiable solution to the quasilinear evolution equation

$$
\begin{aligned}
& u^{\prime}(t)+A(u) u(t)=0, \quad 0<t \leq a, \\
& u(0)=u_{0}
\end{aligned}
$$

under the assumptions similar to one considered by Kato [14]. Pazy [21] considered the following quasilinear equation

$$
\begin{aligned}
& u^{\prime}(t)+A(t, u) u(t)=0, \quad 0<t \leq a, \\
& u(0)=u_{0}
\end{aligned}
$$

and discussed the mild and classical solutions by using a fixed point argument. The same problem has been studied to the nonhomogeneous quasilinear evolution equation

$$
\begin{aligned}
& u^{\prime}(t)+A(t, u) u(t)=f(t, u), \quad 0<t \leq a, \\
& u(0)=u_{0}
\end{aligned}
$$

Received August 29, 2008.

2000 Mathematics Subject Classification. 34G20, 47H20.

Key words and phrases. existence of solution, quasilinear integrodifferential equation, analytic semigroup, fixed point theorem.

This paper is supported by Dong-A University Research Fund 2006.

(C)2009 The Korean Mathematical Society 
by Furuya [10], Kato [13], Sobolevski [25] and Yagi [27]. Bahuguna [3] proved the existence, uniqueness and continuous dependance of a strong solution for quasilinear integrodifferential equations of the form

$$
\begin{aligned}
& u^{\prime}(t)+A(t, u) u(t)=\int_{0}^{t} a(t-s) k(s, u(s)) d s+f(t), \quad 0 \leq t \leq a, \\
& u(0)=u_{0}
\end{aligned}
$$

by using the method of lines. He also established a local classical solution for the same equation in [4]. Oka [19] and Oka and Tanaka [20] investigated the existence of classical solutions of abstract quasilinear integrodifferential equations. An equation of this type occurs in a nonlinear conservation law with memory

$$
\begin{aligned}
u_{t}(t, x)+\Psi(u(t, x))_{x} & =\int_{0}^{t} b(t-s) \Psi(u(t, x))_{x} d s+f(t, x), t \in[0, a], x \in \mathbb{R} \\
u(0, x) & =\phi(x), x \in \mathbb{R} .
\end{aligned}
$$

It is interesting to investigate the existence problem for these type of equations in Banach spaces. The aim of this paper is to study the existence of solutions of quasilinear integrodifferential equations in Banach spaces by using fractional powers of operators and the Schauder fixed-point theorem. The results generalize the results of $[4,13,21,25]$.

\section{Preliminaries}

Consider the initial value problem

$$
\begin{aligned}
x^{\prime}(t)+A(t) x(t) & =f(t) \quad 0 \leq s<t \leq a \\
x(s) & =y
\end{aligned}
$$

with the following assumptions:

$\left(P_{1}\right)$ The domain $D(A(t))=D$ of $A(t), 0 \leq t \leq a$ is dense in $X$ and independent of $t$;

$\left(P_{2}\right)$ For $t \in[0, a]$, the resolvent $R(\lambda ; A(t))=(\lambda I-A(t))^{-1}$ of $A(t)$ exists for all $\lambda$ with $\operatorname{Re} \lambda \leq 0$ and there is a constant $C$ such that

$$
\|R(\lambda ; A(t))\| \leq C[|\lambda|+1]^{-1} \text { for } \operatorname{Re} \lambda \leq 0, t \in[0, a] ;
$$

$\left(P_{3}\right)$ There exist constants $L$ and $0<\alpha \leq 1$ such that

$$
\|(A(t)-A(s)) A(\tau)\| \leq L|t-s|^{\alpha} \text { for } t, s, \tau \in[0, a] .
$$

Theorem 2.1. Under the assumptions $\left(P_{1}\right)-\left(P_{3}\right)$ there is a unique evolution system $U(t, s)$ on $0 \leq s \leq t \leq a$, satisfying

(i) $\|U(t, s)\| \leq M_{0}$ for $0 \leq s \leq t \leq a$ 
(ii) For $0 \leq s \leq t \leq a, U(t, s): X \rightarrow D$ and $t \rightarrow U(t, s)$ is strongly differentiable in $X$. The derivative $\frac{\partial}{\partial t} U(t, s) \in B(X)$ and it is strongly continuous on $0 \leq s<t \leq a$. More over,

$$
\begin{gathered}
\frac{\partial}{\partial t} U(t, s)+A(t) U(t, s)=0 \text { for } 0 \leq s<t \leq a \\
\left\|\frac{\partial}{\partial t} U(t, s)\right\|=\|A(t) U(t, s)\| \leq M_{0}(t-s)^{-1}
\end{gathered}
$$

and

$$
\left\|A(t) U(t, s) A^{-1}(s)\right\|<M_{0} \text { for } 0<s<t<a .
$$

(iii) For every $v \in D$ and $t \in[0, a], U(t, s) v$ is differentiable with respect to $s$ on $0 \leq s \leq t \leq a$ and

$$
\frac{\partial}{\partial t} U(t, s) v=U(t, s) A(s) v
$$

Note that $\left(P_{2}\right)$ and the fact that $D$ is dense in $X$ imply that for every $t \in$ $[0, a],-A(t)$ is the infinitesimal generator of an analytic semigroup. We define the classical solutions of (1) as functions $x:[s, a] \rightarrow X$ which are continuous for $s \leq t \leq a$, continuously differentiable for $s<t \leq a, x(t) \in D$ for $s<$ $t \leq a, x(s)=y$ and $x^{\prime}(t)+A(t) x(t)=f(t)$ holds for $s<t \leq a$. We will call a function $x(t)$ a solution of the initial value problem (1) if it is a classical solution of this problem.

Theorem 2.2. Let $A(t), 0 \leq t \leq a$ satisfy the conditions $\left(P_{1}\right)-\left(P_{3}\right)$ and let $U(t, s)$ be the evolution system in Theorem 2.1. If $f$ is Holder continuous on $[0, a]$, then the initial value problem (1) has, for every $y \in X$, a unique solution $x(t)$ given by

$$
x(t)=U(t, s) y+\int_{s}^{t} U(t, \tau) f(\tau) d \tau .
$$

The proofs of the above theorems can be found in $[9,21]$.

Now consider the quasilinear integrodifferential evolution equations of the form

$$
\begin{aligned}
x^{\prime}(t)+A(t, x(t)) x(t) & =f(t, x(t))+\int_{0}^{t} k(t, s) g(s, x(s)) d s, \\
x(0) & =x_{0},
\end{aligned}
$$

where $-A(t, x)$ is the infinitesimal generator of an analytic semigroup in a Banach space $X$. The nonlinear operators $f, g: J \times X \rightarrow X$ are uniformly bounded and continuous in all of its arguments and $k: \Delta \rightarrow J$ is continuous. Here $J=[0, a]$ and $\Delta=\{(t, s): 0 \leq s \leq t \leq a\}$. Throughout the paper $C_{i}$ 's are positive constants.

Let $r>0$ and take $B_{r}=\{y \in X:\|y\|<r\}$, and assume the following conditions: 
(i) The operator $A_{0}=A\left(0, x_{0}\right)$ is a closed operator with domain $D$ dense in $X$ and

$$
\left\|\left(\lambda I-A_{0}\right)^{-1}\right\| \leq C[|\lambda|+1]^{-1}
$$

for all $\lambda$ with $\operatorname{Re} \lambda \leq 0$ and $C>0$.

(ii) The operator $A_{0}^{-1}$ is a completely continuous operator in $X$.

(iii) For some $\alpha \in[0,1)$ and for any $y \in B_{r}$ the operator $A\left(t, A_{0}^{-\alpha} y\right)$ is well defined on $D$ for all $t \in J$. Further more for any $t, \tau \in J$ and for $y, z \in B_{r}$

$\left.\| A\left(t, A_{0}^{-\alpha} y\right)-A\left(\tau, A_{0}^{-\alpha} z\right)\right] A^{-1}\left(\tau, A_{0}^{-\alpha} z\right) \| \leq C_{1}\left[|t-\tau|^{\epsilon}+\|y-z\|^{\rho}\right]$, where $0<\epsilon \leq 1, \quad 0<\rho \leq 1$.

(iv) For every $t, \tau \in J$ and $y, z, \in B_{r}$

$$
\left\|f\left(t, A_{0}^{-\alpha} y\right)-f\left(\tau, A_{0}^{-\alpha} z\right)\right\| \leq C_{2}\left[|t-\tau|^{\epsilon}+\|y-z\|^{\rho}\right] .
$$

(v) For every $t \in J$ and $y, z \in B_{r}$

$$
\left\|g\left(s, A_{0}^{-\alpha} y\right)-g\left(s, A_{0}^{-\alpha} z\right)\right\| \leq C_{3}\|y-z\|^{\rho} .
$$

(vi) For every $t, s, \tau \in J$

$$
|k(t, s)-k(\tau, s)| \leq C_{4}|t-\tau|^{\epsilon} .
$$

(vii) $x_{0} \in D\left(A_{0}^{\beta}\right)$ for some $\beta>\alpha$ and

$$
\left\|A_{0}^{\alpha} x_{0}\right\|<r .
$$

\section{Main result}

Theorem 3.1. If the hypotheses (i)-(vii) are satisfied, then there exists at least one continuously differentiable solution of the equation (3) on $(0, T]$ for some $T \leq a$.

Proof. In order to study the existence problem, we must introduce a set $S$ of functions $x(t), t \in[0, T]$ and a transformation $z_{x}=\Phi x$ defined by $z_{x}=A_{0}^{\alpha} z$, where $z$ is the unique solution of

$$
\begin{aligned}
\frac{d z}{d t}+A_{x}(t) z & =f\left(t, A_{0}^{-\alpha} x(t)\right)+\int_{0}^{t} k(t, s) g\left(s, A_{0}^{-\alpha} x(s)\right) d s, \\
z(0) & =x_{0} .
\end{aligned}
$$

We then show that $\Phi$ has a fixed point, that is, there is a function $y \in S$ such that $\Phi y=y$, and so $x=A_{0}^{-\alpha} y$ is the required solution of our problem (3). Define the set

$$
S=\left\{x \in Y:\|x(t)-x(\tau)\| \leq K|t-\tau|^{\eta} \text { for } t, \tau \in[0, T], x(0)=A_{0}^{\alpha} x_{0}\right\},
$$

where $K$ is a positive constant and $\eta$ is any number satisfying $0<\eta<\beta-\alpha$ and $Y$ is a Banach space $C(J, X)$ with usual supnorm. From hypothesis (vii), and the definition of $S$ it follows that if $T$ is sufficiently small (depending on $\left.K, \eta,\left\|A_{0}^{\alpha} x_{0}\right\|\right)$, then

$$
\|x(t)\|<r \text { for } t \in[0, T] .
$$


Hence the operator $A_{x}(t)=A\left(t, A_{0}^{\alpha} x(t)\right)$ is well defined and satisfies the conditions

$$
\begin{aligned}
\left\|\left(A_{x}(t)-A_{x}(\tau)\right) A_{0}^{-1}\right\| & \leq C_{5}\left[|t-\tau|^{\epsilon}+\|x(t)-x(\tau)\|^{\rho}\right] \\
& \leq C_{6}|t-\tau|^{\mu}
\end{aligned}
$$

where $\mu=\min \{\epsilon, \rho \eta\}$. Further, if $x(0)=A_{0}^{\alpha} x_{0}$,

$$
A_{x}(0)=A\left(0, A_{0}^{-\alpha} x(0)\right)=A\left(0, A_{0}^{-\alpha} A_{0}^{\alpha} x_{0}\right)=A\left(0, x_{0}\right)=A_{0},
$$

and it follows that for every $t \in[0, T]$ and $\lambda$ with $\operatorname{Re} \lambda \leq 0$

$$
\begin{aligned}
\left\|\left[\lambda I-A_{x}(t)\right]^{-1}\right\| & \leq C_{7}[|\lambda|+1]^{-1}, \\
\left\|\left[A_{x}(t)-A_{x}(\tau)\right] A_{x}^{-1}(s)\right\| & \leq C_{8}|t-\tau|^{\mu} \text { for any } t, \tau, s \in[0, T] .
\end{aligned}
$$

By the hypotheses (i)-(iii) there exists a fundamental solution $U_{x}(t, s)$ corresponding to $A_{x}(t)$, and all estimates for fundamental solutions derived in Theorem 2.1 hold uniformly with respect to $x \in S$. From our assumptions, we have

$$
\left\|A_{0}^{\alpha}\left[U_{x}\left(t_{1}, 0\right)-U_{x}\left(t_{2}, 0\right)\right] A_{0}^{-\beta}\right\| \leq C_{9}\left|t_{1}-t_{2}\right|^{\beta-\alpha} .
$$

From (v) and (vi), we can see that there exist constants $M_{1}>0, M_{2}>0$ such that

Let us take

$$
\left\|g\left(t, A_{0}^{-\alpha} x(t)\right)\right\| \leq M_{1} \text { and }|k(t, s)| \leq M_{2} .
$$

$$
f_{x}(t)=f\left(t, A_{0}^{-\alpha} x(t)\right), \quad g_{x}(t)=\int_{0}^{t} k(t, s) g\left(s, A_{0}^{-\alpha} x(s)\right) d s .
$$

Then, it follows that the function $f_{x}(t)$ is Holder continuous such that

$$
\left\|f_{x}(t)-f_{x}(\tau)\right\| \leq C_{10}|t-\tau|^{\mu}, \quad\left\|g_{x}(t)-g_{x}(\tau)\right\| \leq C_{11}|t-\tau|^{\mu} .
$$

Since $f_{x}(0)=f\left(0, A_{0}^{-\alpha} x(0)\right)$ and $g_{x}(0)=0$ are independent of $x$, we have from the above inequalities

$$
\left\|f_{x}(t)\right\| \leq M_{3}, \quad\left\|g_{x}(t)\right\| \leq M_{4}, \quad M_{3}>0, \quad M_{4}>0
$$

and

$$
\begin{aligned}
& \left\|A_{0}^{\alpha}\left[\int_{0}^{t_{1}} U_{x}\left(t_{1}, s\right)\left(f_{x}(s)+g_{x}(s)\right) d s-\int_{0}^{t_{2}} U_{x}\left(t_{2}, s\right)\left(f_{x}(s)+g_{x}(s)\right) d s\right]\right\| \\
\leq & C_{12}\left|t_{1}-t_{2}\right|^{1-\alpha} .
\end{aligned}
$$

We shall show that the operator $\Phi: S \rightarrow Y$ defined by

$$
\Phi x(t)=A_{0}^{\alpha} U_{x}(t, 0) x_{0}+A_{0}^{\alpha} \int_{0}^{t} U_{x}(t, s)\left[f_{x}(s)+g_{x}(s)\right] d s
$$

has a fixed point. This fixed point is the solution of equation (3). Clearly $S$ is closed convex and bounded subset of $Y$. First we show that $\Phi$ maps $S$ into itself. Obviously $\Phi x(0)=A_{0}^{\alpha} x_{0}$. 
For any $0 \leq \alpha<\beta \leq 1$ and $0 \leq t_{1} \leq t_{2} \leq T$, we have

$$
\begin{aligned}
& \left\|\Phi x\left(t_{1}\right)-\Phi x\left(t_{2}\right)\right\| \\
\leq & \left\|A_{0}^{\alpha}\left[U_{x}\left(t_{1}, 0\right)-U_{x}\left(t_{2}, 0\right)\right] A_{0}^{-\beta}\right\|\left\|A_{0}^{\beta} x_{0}\right\| \\
& +\left\|A_{0}^{\alpha} \int_{0}^{t_{1}} U_{x}\left(t_{1}, s\right)\left[f_{x}(s)+g_{x}(s)\right] d s-A_{0}^{\alpha} \int_{0}^{t_{2}} U_{x}\left(t_{2}, s\right)\left[f_{x}(s)+g_{x}(s)\right] d s\right\| .
\end{aligned}
$$

Thus, for $T$ sufficiently small,

$$
\begin{aligned}
\left\|\Phi x\left(t_{1}\right)-\Phi x\left(t_{2}\right)\right\| & \leq r C_{9}\left|t_{1}-t_{2}\right|^{\beta-\alpha}+C_{12}\left|t_{1}-t_{2}\right|^{1-\alpha} \\
& \leq K\left|t_{1}-t_{2}\right|^{\eta} \text { for some } K>0, \eta<\beta-\alpha .
\end{aligned}
$$

Hence $\Phi$ maps $S$ into itself.

Next we show that this operator is continuous on the space $Y$. Let $x_{1}, x_{2} \in S$ and set $z_{1}=A_{0}^{-\alpha} \Phi x_{1}, z_{2}=A_{0}^{-\alpha} \Phi x_{2}$. Then,

$$
\begin{aligned}
\frac{d z_{i}}{d t}+A_{x_{i}}(t) z_{i} & =f_{x_{i}}(t)+g_{x_{i}}(s) \\
z_{i}(0) & =x_{0}, i=1,2 .
\end{aligned}
$$

Therefore,

$$
\begin{aligned}
& \frac{d}{d t}\left(z_{1}-z_{2}\right)+A_{x_{1}}(t)\left(z_{1}-z_{2}\right) \\
= & {\left[A_{x_{2}}(t)-A_{x_{1}}(t)\right] z_{2}+f_{x_{1}}(t)-f_{x_{2}}(t)+g_{x_{1}}(t)-g_{x_{2}}(t) . }
\end{aligned}
$$

It is easy to see that the functions $A_{x_{2}}(t) z_{2}(t)$ and $A_{0} A_{x_{2}}^{-1}(t)$ are uniformly Holder continuous, and so $A_{0} z_{2}(t)=\left[A_{0} A_{x_{2}}^{-1}(t)\right] A_{x_{2}}(t) z_{2}(t)$ is uniformly Holder continuous. Similarly the functions

$$
f_{x_{1}}(t)-f_{x_{2}}(t), g_{x_{1}}(t)-g_{x_{2}}(t)
$$

are also uniformly Holder continuous in $[\tau, T], \tau>0$. Hence, we have

$$
\begin{aligned}
& {\left[z_{1}(t)-z_{2}(t)\right] } \\
= & U_{x_{1}}(t, \tau)\left[z_{1}(\tau)-z_{2}(\tau)\right]+\int_{0}^{t} U_{x_{1}}(t, s)\left(\left[A_{x_{2}}(s)-A_{x_{1}}(s)\right] z_{2}(s)\right. \\
& \left.+\left[f_{x_{1}}(s)-f_{x_{2}}(s)\right]+\left[g_{x_{1}}(s)-g_{x_{2}}(s)\right]\right) d s .
\end{aligned}
$$

Since $A_{0} \int_{0}^{t} U_{x_{2}}(t, s)\left[f_{x_{2}}(s)+g_{x_{2}}(s)\right] d s$ is a bounded function, it follows that

$$
\left\|A_{0} z_{2}(t)\right\| \leq C_{13} t^{\beta-1} \text {. }
$$

Hence we can take $\tau \rightarrow 0$ in the above equation and we get

$$
\begin{aligned}
{\left[z_{1}(t)-z_{2}(t)\right]=} & \int_{0}^{t} U_{x_{1}}(t, s)\left(\left[A_{x_{2}}(s)-A_{x_{1}}(s)\right] z_{2}(s)\right. \\
& \left.+\left[f_{x_{1}}(s)-f_{x_{2}}(s)\right]+\left[g_{x_{1}}(s)-g_{x_{2}}(s)\right]\right) d s .
\end{aligned}
$$


Since $z_{1}=A_{0}^{-\alpha} \Phi x_{1}$ and $z_{2}=A_{0}^{-\alpha} \Phi x_{2}$ and from (iii), (iv), (v) and (vi) it follows that

$$
\begin{aligned}
\left\|\Phi x_{1}(t)-\Phi x_{2}(t)\right\| \leq & \int_{0}^{t}\left\|A_{0}^{\alpha} U_{x_{1}}(t, s)\right\|\left[\left\|\left[A_{x_{2}}(s)-A_{x_{1}}(s)\right] z_{2}(s)\right\|\right. \\
& \left.+\left\|f_{x_{1}}(s)-f_{x_{2}}(s)\right\|+\left\|g_{x_{1}}(s)-g_{x_{2}}(s)\right\|\right] d s \\
\leq & \int_{0}^{t} C_{14}|t-s|^{-\alpha}\left[C_{15}\left\|x_{1}(s)-x_{2}(s)\right\|^{\rho} s^{\beta-1}\right. \\
& \left.+C_{16}\left\|x_{1}(s)-x_{2}(s)\right\|^{\rho}\right] d s .
\end{aligned}
$$

Hence

$$
\left\|\Phi x_{1}-\Phi x_{2}\right\|_{Y} \leq K^{*} T^{\beta-\alpha}\left\|x_{1}-x_{2}\right\|_{Y}^{\rho} \text { for some } K^{*}>0
$$

This shows that $\Phi: S \rightarrow Y$ is continuous. We shall show that this operator is completely continuous. We now claim that the set $\Phi S$ is contained in a compact subset of $Y$. Indeed, the functions $x(t)$ of $S$ are uniformly bounded and equicontinuous. By Arzela-Ascoli's theorem it is sufficient to show that for each $t$ the set $\{\Phi x(t) ; \quad x \in S\}$ is contained in a compact subset of $X$. For each $t \in[0, T]$, we can write $\Phi x(t)=A_{0}^{-\gamma} A_{0}^{\gamma} \Phi x(t),(0<\gamma<\beta-\alpha)$. Since $\left\{A_{0}^{\gamma} \Phi x(t): x \in S\right\}$ is a bounded subset of $X$, and since $A_{0}^{-\gamma}$ is completely continuous, it follows that the set $\{\Phi x(t): x \in S\}$ is contained in a compact subset of $X$. Therefore by the Schauder fixed point theorem, $\Phi$ has a fixed point $z \in S$ such that $\Phi z(t)=z(t)$ which satisfies

$$
z(t)=A_{0}^{\alpha} U_{z}(t, 0) x_{0}+A_{0}^{\alpha} \int_{0}^{t} U_{z}(t, s)\left[f_{z}(s)+g_{z}(s)\right] d s .
$$

Then $x(t)=A_{0}^{-\alpha} z(t)$ satisfies

$$
x(t)=U_{A_{0}^{\alpha} x}(t, 0) x_{0}+\int_{0}^{t} U_{A_{0}^{\alpha} x}(t, s)\left[f_{A_{0}^{\alpha} x}(s)+g_{A_{0}^{\alpha} x}(s)\right] d s .
$$

By Theorem 2.2, $x(t)$ is a solution of (3).

Theorem 3.2. Let the assumptions (i), (iii)-(v) hold with $\rho=1$. Then the assertion of Theorem 3.1 is valid and the solution is unique.

Proof. If $\rho=1$, then from (6) shows that for $T$ sufficiently small $\Phi$ is a contraction, that is $\left\|\Phi x_{1}-\Phi x_{2}\right\| \leq \theta\left\|x_{1}-x_{2}\right\|$ for some $\theta<1$. Hence by the Banach fixed point theorem $\Phi$ has a unique fixed point. 


\section{Example}

Consider the following nonlinear parabolic integrodifferential equation

$$
\begin{aligned}
& \frac{\partial z}{\partial t}+\Sigma_{|\alpha|=2 m} a_{\alpha}\left(x, t, z, D z, \ldots, D^{2 m-1} z\right) D^{\alpha} z \\
= & f\left(x, t, z, D z, \ldots, D^{2 m-1} z\right)+\int_{0}^{t} k(x, t, s) g\left(x, s, z, D z, \ldots, D^{2 m-1} z\right) d s, \\
\frac{\partial^{j} z}{\partial \nu^{j}}= & 0 \text { on } S_{T}=\{(x, t): x \in \partial \Omega, 0 \leq t \leq T\}, 0 \leq j \leq m-1 \\
u(x, 0)= & 0 \text { on } \Omega_{0}=\{(x, 0): x \in \partial \Omega\}
\end{aligned}
$$

in a cylinder $Q_{T}=\Omega \times(0, T)$ with coefficients in $\bar{Q}_{T}$, where $\Omega$ is a bounded domain in $R^{n}, \partial \Omega$ the boundary of $\Omega, \nu$ is the outward normal. Here the parabolicity means that for any vector $y \neq 0$ and for arbitrary values of $z, D z, \ldots, D^{2 m-1} z$,

$$
(-1)^{m} \operatorname{Re}\left\{\Sigma_{|\alpha|=2 m} a_{\alpha}\left(x, t, z, D z, \ldots, D^{2 m-1} z\right) y^{\alpha}\right\} \geq C|y|^{2 m}, C>0 .
$$

If $z_{0}(x) \in C^{2 m-1}(\bar{\Omega})$, then

$$
A_{0} z=\Sigma_{|\alpha|=2 m} a_{\alpha}\left(x, t, z, D z, \ldots, D^{2 m-1} z\right) D^{\alpha} z
$$

is a strongly elliptic operator with continuous coefficients. So the condition (i) holds. Let us take $X$ to be $L^{p}(\Omega), 1<p<\infty$. Then $A_{0}^{-1}$ maps bounded subsets of $L^{p}(\Omega)$ in to bounded subsets of $W^{2 m, p}(\Omega)$, so it is a completely continuous operator in $L^{p}(\Omega)$. Further, if $(2 m-1) / 2 m<\alpha<1$, then [9]

$$
\left|D^{\beta} A_{0}-\alpha z\right|_{0, p}^{\Omega} \leq C|z|_{0, p}^{\Omega}, \quad 0 \leq|\beta| \leq 2 m-1,
$$

where $C$ depends only on a bound on the coefficients $A_{0}$, on a module of strong ellipticity and on a modulus of continuity of the leading coefficients. Here the norm is defined as

$$
|z|_{j, p}^{\Omega}=\left\{\sum_{|\alpha| \leq j} \int_{\Omega}\left|D^{\alpha} z(x)\right|^{p} d x\right\}^{\frac{1}{p}}
$$

for any nonnegative integer $j$ and a real number $p, 1 \leq p<\infty$. It follows that if $f$ and $a_{\alpha}$ are continuously differentiable in all variables, then (iii) and (iv) hold with $\sigma=\rho=1$. Hence there exist fundamental operator solution $U_{x}(t, s)$ for the equation (7). The nonlinear functions $f, g$ satisfy the conditions (iv),(v) and $k$ satisfies the condition (vi). Hence by the above theorem there exist a local solution for the equation (7).

\section{References}

[1] H. Amann, Quasilinear evolution equations and parabolic systems, Trans. Amer. Math. Soc. 293 (1986), no. 1, 191-227.

[2] E. H. Anderson, M. J. Anderson, and W. T. England, Nonhomogeneous quasilinear evolution equations, J. Integral Equations 3 (1981), no. 2, 175-184. 
[3] D. Bahuguna, Quasilinear integrodifferential equations in Banach spaces, Nonlinear Anal. 24 (1995), no. 2, 175-183.

[4] _ Regular solutions to quasilinear integrodifferential equations in Banach spaces, Appl. Anal. 62 (1996), no. 1-2, 1-9.

[5] K. Balachandran and K. Uchiyama, Existence of solutions of quasilinear integrodifferential equations with nonlocal condition, Tokyo J. Math. 23 (2000), no. 1, 203-210.

[6] _ Existence of local solutions of quasilinear integrodifferential equations in Banach spaces, Appl. Anal. 76 (2000), no. 1-2, 1-8.

[7] F. Colombo, Quasilinear parabolic equations in $C^{k}$ spaces, Dynam. Systems Appl. 6 (1997), no. 2, 271-296.

[8] M. G. Crandall and P. E. Souganidis, Convergence of difference approximations of quasilinear evolution equations, Nonlinear Anal. 10 (1986), no. 5, 425-445.

[9] A. Friedman, Partial Differential Equations, Holt, Rinehart and Winston, Inc., New York, 1969.

[10] K. Furuya, Analyticity of solutions of quasilinear evolution equations. II, Osaka J. Math. 20 (1983), no. 1, 217-236.

[11] R. Ikehata and N. Okazawa, A class of second order quasilinear evolution equations, J. Differential Equations 114 (1994), no. 1, 106-131.

[12] A. G. Kartsatos, Perturbations of $M$-accretive operators and quasi-linear evolution equations, J. Math. Soc. Japan 30 (1978), no. 1, 75-84.

[13] S. Kato, Nonhomogeneous quasilinear evolution equations in Banach spaces, Nonlinear Anal. 9 (1985), no. 10, 1061-1071.

[14] T. Kato, Quasi-linear equations of evolution, with applications to partial differential equations, Spectral theory and differential equations (Proc. Sympos., Dundee, 1974; dedicated to Konrad Jorgens), pp. 25-70. Lecture Notes in Math., Vol. 448, Springer, Berlin, 1975.

[15] Abstract evolution equations, linear and quasilinear, revisited, Functional analysis and related topics, 1991 (Kyoto), 103-125, Lecture Notes in Math., 1540, Springer, Berlin, 1993.

[16] K. Kobayasi and N. Sanekata, A method of iterations for quasi-linear evolution equations in nonreflexive Banach spaces, Hiroshima Math. J. 19 (1989), no. 3, 521-540.

[17] A. Lunardi, Global solutions of abstract quasilinear parabolic equations, J. Differential Equations 58 (1985), no. 2, 228-242.

[18] M. G. Murphy, Quasilinear evolution equations in Banach spaces, Trans. Amer. Math. Soc. 259 (1980), no. 2, 547-557.

[19] H. Oka, Abstract quasilinear Volterra integrodifferential equations, Nonlinear Anal. 28 (1997), no. 6, 1019-1045.

[20] H. Oka and N. Tanaka, Abstract quasilinear integrodifferential equations of hyperbolic type, Nonlinear Anal. 29 (1997), no. 8, 903-925.

[21] A. Pazy, Semigroups of Linear Operators and Applications to Partial Differential Equations, Springer-Verlag, New York, 1983.

[22] N. Sanekata, Convergence of approximate solutions to quasilinear evolution equations in Banach spaces, Proc. Japan Acad. Ser. A Math. Sci. 55 (1979), no. 7, 245-249.

[23] - Abstract quasi-linear equations of evolution in nonreflexive Banach spaces, Hiroshima Math. J. 19 (1989), no. 1, 109-139.

[24] _ Abstract quasi-linear equations of evolution with application to first order quasi-linear hyperbolic systems in two independent variables, Adv. Math. Sci. Appl. 3 (1993/94), Special Issue, 119-159.

[25] P. E. Sobolevskii, Equations of parabolic type in Banach space, Amer. Math. Soc. Transl. 49 (1965), 1-62.

[26] N. Tanaka, Quasilinear evolution equations with non-densely defined operators, Differential Integral Equations 9 (1996), no. 5, 1067-1106. 
[27] A. Yagi, Abstract quasilinear evolution equations of parabolic type in Banach spaces, Boll. Un. Mat. Ital. B (7) 5 (1991), no. 2, 341-368.

KRISHNAN BALACHANDRAN

Department of Mathematics

BHARATHIAR UNIVERSITY

Coimbatore 641 046, India

E-mail address: balachandran-k@lycos.com

DONG Gun PARK

Department of Mathematics

DONG-A University

Pusan 604-714, Korea

E-mail address: dgpark@dau.ac.kr 